\title{
Harnessing the Sars-cov-2 Spike Protein Binding Affinity to Ace2 Receptor Through Narcotinic Compounds Combined With Adjuvants: an in Silico Insight
}

\section{Saeedeh Mohammadi ( $\nabla$ s.mohammadi@sru.ac.ir)}

Shahid Rajaee Teacher Training University

\section{Esmail Doustkhah}

National Institute for Materials Science

Nader Sakhaee

University of Illinois at Urbana Champaign

Ayoub Esmailpour

Shahid Rajaee Teacher Training University

Mohammad Esmailpour

Azarbaijan Shahid Madani University

\section{Research Article}

Keywords: Narcotic compounds, ACE2 human receptors, Inhibitor, Adjuvants, Drug repurposing

Posted Date: March 4th, 2021

DOI: https://doi.org/10.21203/rs.3.rs-254891/v1

License: (c) (i) This work is licensed under a Creative Commons Attribution 4.0 International License.

Read Full License 


\section{Abstract}

Protein products of SARS-CoV-2 spike (S) coding gene sequence, were all analyzed and compared to other SARS-CoV S proteins to elucidate structural similarities of spike proteins. A homology modeling of SARS-CoV-2 S protein was obtained and used in molecular docking studies to find binding affinities of spike protein for angiotensin-converting enzyme 2 (ACE2). The two most important binding sites of $S$ protein, namely, RBD and CTD, critically responsible for binding interactions, were identified. Finally, binding affinity of RBD and CTD domains of S protein with narcotic analgesics are studied. Moreover, interactions of ACE2 receptor-S protein with narcotic compounds when mixed with small molecule adjuvants to improve the immune response and increase the efficacy of potential vaccines, were taken into consideration. In-silico results suggest that the combination of narcotine hemiacetal with mannide monooleate shows a stronger binding affinity with CTD, while carprofen-muramyl dipeptide and squalene have stronger binding affinities for the RBD portion of S protein. Thus, a suitable combination of these narcotic is proposed to yield potent site-blocking efficacy for ACE2 receptor against SARS-CoV-2 spike proteins.

\section{Introduction}

Recently emerged novel coronavirus (2019-nCoV) originated in Wuhan, China [1] is currently the most significant ongoing threat to human health worldwide [2,3]. Severe acute respiratory syndrome coronavirus 2 (SARS-CoV-2) is the third highly pathogenic pneumonia coronavirus after SARS-CoV-1 and MERS-CoV (middle east respiratory syndrome-related coronavirus) to cause a full-blown pandemic [4,5]. According to the World Health Organization (WHO), by 17 February 2021, over 109 million infection cases globally and almost 2,413,000 deaths have been reported [6]. To inhibiting SARS-CoV-2, there are potentially effective strategies such as the design of an effective vaccine and the discovery of a potential drug that capable target active sites of SASR-CoV-2 [7-12]. Meanwhile, how the virus enters a host cell has a significant effect to develop a novel drug and/or repurpose existing drugs.

Angiotensin-converting enzyme 2 (ACE2) contributes to viral cell entry through the interaction with S protein [13-15]. Finding a compound that can block the formation of the complex between the SARS-CoV$2 \mathrm{~S}$ protein receptor binding domain (RBD) and the ACE2 receptor or disrupt in RBD-ACE2 complex has been followed as a reasonable strategy to come up with a rational drug discovery for COVID-19 [16]. Since the development or discovery of a novel drug is often costly and might take several years [17], repurposing a drug for timely release to the market significantly lowers the cost and help fight the pandemic in its most aggressive phases. Although several efforts are underway, there is so far no promising antiviral agent for SARS-CoV-2 in final quarter of 2020. Therefore, we still need to review existing drugs in order to discover their antiviral properties [18].

Narcotic compounds are bioactive natural alkaloids mostly with plant origins which act as stimulants and inhibitors in the biological system of mammals including humans. Although use of high-dose narcotic analgesics such as morphine and fentanyl have been observed to cause immune suppression, 
they are safe, economic, and effective for the management of severe cancer pain when used by medical advice and with precautions [19-21]. Recent studies suggest that narcotic compounds such as fentanyl and lidocaine can minimize aerosolization risk of coughing during extubation [22-27]. In China, a preliminary analysis in a case study report, examined data from 5 case series of hospitalized COVID-19 patients, and calculated a smoking prevalence of $10.2 \%$ (95\% Cl: 8.7-11.8 \%) while the estimated expected prevalence was $31.3 \%$ (95\% Cl: 8.7-11.8\%) [28]. The analysis was further expanded well into the second pandemic wave, by examining 13 Chinese studies and 5960 hospitalized COVID-19 patients, with a pooled smoking prevalence of $6.5 \%$ (95\% Cl: 4.9-8.2 \%) [29]. This suggests a hypothesis that nicotine may be protective against severe COVID-19, which is biologically plausible and should be further investigated in clinical trials on nicotine as a drug candidate [30]. These clinical trials should test the effects of the smoking and nicotine on the risk of being infected with COVID-19 (NCT04429815). We recently reported combinations, i.e., nicotine and caffeine, for blocking ACE2 receptor against SARS-CoV-2 [31]. In this paper, we investigated for the first time a hypothesis about the potential benefits of narcotic analgesics using in delivery systems as an adjuvant (i.e., Montanide ISA 720, muramyl dipeptide, squalene and Adjuvant Systems 01 (AS01)) to improve the immune response and increase the efficacy of vaccines. We hypothesized that there could be a potential S protein-ACE2 site-blocking activity through forming a complex between narcotic compounds with small molecule adjuvants.

\section{Methods}

The interactions of narcotic compounds with the SARS-CoV-2 S protein- ACE2 compared to SARS-CoV S protein with ACE2 complex were investigated. The chemical structure backbones of narcotic compounds resemble some anti-COVID-19 drug candidates. Therefore, we decided to investigate the possible interactions and potential blocking activity of these narcotic compounds with the CTD/RBD-ACE2. Such interaction sites are the epitopes of S protein and the corresponding ACE2 receptor responsible for COVID19 cell binding mechanisms. This blocking behavior is intensified when the compounds are mixed with adjuvants such as Montanide ISA 720, muramyl dipeptide, squalene and AS01. Our focus will be directed toward the potency of narcotic compounds acting as COVID-19 antiviral drugs by blocking the ACE2 receptor mechanism.

\section{Selected Narcotic Compounds}

Several narcotic compounds have been introduced in treatment of human immunodeficiency virus (HIV), SARS-CoV and COVID-19. Accordingly, we've selected twenty narcotic analgesics as presented in Figure 1. The interaction of narcotic analgesics with the RBD/CTD-ACE2 receptor when combined with these adjuvants were investigated to study their inhibition mechanism as well as their efficacy as potential drug candidates.

\section{Selected small molecule adjuvants}

Available vaccines can induce weak immunogenicity and often do not conduce an effective immune response [32]. An underexplored approach for maximising the efficacy, efficiency and enduring effect of 
vaccines is to discover, develop and optimize efficacious adjuvants [33-35]. Adjuvants can be used by enhancing antigen presentation to antigen-specific immune cells in order to both improve immunogenicity and conduce long-term protection against pathogens [35]. Adjuvants can range in their chemical structure from proteins, complex natural products, oligonucleotides, drug-like small molecules to certain delivery systems, such as those based on liposomes, which possess intrinsic adjuvant activity [34]. Here four selected adjuvants are discussed, see Figure 2.

\subsection{Montanide ISA 720}

Oil adjuvants, based on non-mineral oil such as Squalene which is a natural product with animal or plant origin, has been also used in human clinical trials [36]. Recently water in oil adjuvant based on squalene like Montanide ISA 720 that contains a mannide monooleate emulsifier [37], are tested in various clinical trial representing more than 500 patients and 1500 injections [36]. Many studies with oil-based adjuvant like Montanide ISA 720 reported the use of these adjuvants for immunotherapy such as HIV [38-40] treatment and even for prophylactic vaccine where no relevant treatment exists like malaria [41-43].

\subsection{Muramyl Dipeptide}

It is found that adjuvants based on peptidoglycan constituents, known as muramyl dipeptide (MDP) can be used to modulate the immune response [44]. It was reported that MDP and their derivatives confer their adjuvanticity effect by activating the NF-KB pathway through the NOD2 receptor [45]. In an in silico study on mices rapid identification of SARS-CoV derived antigenic peptides was observed due to recognition mechanisms by HLA-A2-restricted cytotoxic T lymphocytes (CTLs). It was suggested that muramyl dipeptide can conduce upregulation of HLA-DR, CD80, CD86, and CD40 in human CD14+antigen presenting cells, and it was administered as an adjuvant [46]. It is also proposed that a synthetic vaccine might be designed based on T-epitopes as haptens (for cell response and immune system memory), molecular adjuvant (e.g., muramyl dipeptide), and possibly excitatory or anti-inhibitory peptides for SARSCoV-2 [47].

\section{Homology modeling of SARS-CoV-2 S protein}

All genomic sequences of SARS-CoV-2 S protein (YP_009724390.1) was obtained from National Center for Biotechnology Information (NCBI) nucleotide database. The nucleotide sequences were aligned with whole database using BLASTn to search for homology viral genomes. Accession numbers of 50 sequences in GenBank are listed as follows: QKY78084.1, QLI51781.1, QIU81585.1, QMS52716.1, QKU28894.1, QIS61254.1, QIS60906.1, QLJ57383.1, QIU80973.1, QMI94525.1, QJC19455.1, QMI90807.1, QKV38208.1, QIU81885.1, QMX86773.1, QLC91196.1, QJS39579.1, QNO91835.1, QMT96172.1, QIS61422.1, QJE38426.1, QKV35819.1, QIS30335.1, QKK14611.1, QLC48052.1, QOF12353.1, QJQ84843.1, QNA39510.1, QJF75467.1, QKU32813.1, QJR84873.1, QJR84837.1 etc. it is shown by details in Figure $\mathrm{S} 1$. 
To find possible sites of positive or negative selection Adaptive Evolution Server was used (http://www.datamonkey.org/). Statistically significant positive or negative selection was based on $\mathrm{p}$ value $<0.05[48]$.

Recently, the two crystal structures of the complex between SARS-CoV-2 S protein and ACE2 receptor (e.g., 6LZG, 6VW1) are resolved by X-ray diffraction and cryoelectron microscopy (cryo-EM). According to reported structures for ACE2 and SARS-CoV-2 complex, there are two active sites in SARS-CoV-2 S protein, interacting with ACE2. The SARS-CoV-2 RBD and CTD structures as active sites of the SARS-CoV$2 \mathrm{~S}$ protein were selected in this study to further investigate the binding mechanism to ACE2 receptor. The nucleotide sequence editing was conducted using Bioedit program v7.0.5 [49], and sequence has been aligned using ClustaIW [50]. The evolutionary history was inferred using the Neighbor-Joining method in molecular evolutionary genetics analysis version X (MEGA-X) [51] software package. The percentage of replicate trees in which the associated taxa clustered together in the bootstrap test was determined by 500 replicates. Phylogenetic tree was generated with Jones-Taylor-Thornton (JTT) evolutionary model. Protein homology modelling has been attempted using the website Swiss-Model [52]. Each model was then individually superimposed over the template and root mean square deviation (RMSD) was estimated in Å using SWISS-PdBViewer 4.1.0 [53]. Three dimensional structures have been analyzed and displayed using PyMOL [54].

\section{Molecular docking}

It is suggested that a fast and economical tool (such as molecular docking) can be combined with molecular dynamic simulations (high precision but time consuming) to efficiently conduct more reliable and highly precise calculations in protein-ligand complexes. Moreover, for the fast screening of large libraries, docking can be efficiently utilized to explore the conformations of the protein receptors, optimize the structures of the final complexes, and finally to calculate accurate energies [55].

For targeting the interface of the S protein (in SARS-CoV-2) and the ACE2 receptor by a blocking agent, we evaluated the blocking potential of narcotic compounds, and their combined forms with adjuvants. All the narcotic molecules (www.drugbank.ca/) and adjuvants were energy minimized using Steepest descent algorithm employed in Avogadro. Reported crystal structures in Protein Data Bank (PDB) for ACE2 (PDB: 1R42), SARS-CoV (PDB: 2AJF) and SARS-CoV-2 CTD/RBD encoded were used. All water molecules, ligands and ions were removed in these crystal structures and finally hydrogen atoms were enhanced to better serve the purpose of this study. Binding affinity estimation for the interactions between the $S$ protein RBD/CTD-ACE2 and narcotic compounds mixed with adjuvants mentioned were all performed via molecular docking.

We used the AutoDock v4.2 package for the docking studies [56, 57]. Also, the charges of the molecules were applied. We selected a $60 \times 60 \times 60 \AA$ grid box, and the distance between two grid points was set at $1.0 \AA$ centering on the structures. In this paper, the rigid structure of the proteins was considered, so that, in this state, the drug is assumed to be fixed in shape. By using the Lamarckian genetic algorithm (LGA) [58], we performed molecular docking. In molecular docking through genetic algorithms (GA) [59], the 
particular arrangement of a ligand and a protein can be defined by a set of values describing the translation, orientation, and conformation of the ligand with respect to the protein. All thigh profiles were produced under the following conditions: an initial population of 150 randomly placed individuals and a maximum number of $2.5 \times 106$ energy evaluations, a maximum number of 27,000 generations, a mutation rate of 0.02 , a crossover rate of 0.80 , and an elitism value of 1 . Finally, results were clustered and analyzed considering binding energies and main interacting residues in each complex by bioinformatics module of ICM 3.7.3 modeling software on an Intel i7 4960 processor (MolSoft LLC, San Diego, CA, USA) [60].

\section{Results}

Here, we present our in silico results for calculated binding affinities of narcotic compounds mixed with adjuvants to target proteins such as RBD-ACE2 and CTD-ACE2. Also, we highlight ligands of narcotic compounds mixed with adjuvants that we believe may be targeting the binding between $S$ protein and ACE2, and thus are of special interest for experimental evaluation. A structural representation of the interaction between ACE2/SARS-CoV-2-CTD and SARS-CoV RBD with narcotine hemiacetal binding to mannide monooleate is shown in Figure 3.

Any small molecule bound to $S$ protein at this time may interfere the re-folding of $S$ protein, therefore inhibits the viral infection process. Furthermore, small molecule that can target any part of $S$ protein may be a good starting point to design PROTAC based therapy [61]. For mannide monooleate, we found SARSCoV-2 CTD-ACE2+ narcotine hemiacetal and codeine could be helpful for viral infection treatment, whereas fentanyl is the best option for SARS-CoV RBD-ACE2, as illustrated in Figure 4. Based on our results for strongest binding affinity, initial repurposing may be better suited to carprofen mixed with muramyl dipeptide for both of RBD-ACE2 of SARS-CoV-2 and SARS-CoV. Also, the most efficient compounds are narcotine hemiacetal with SARS-CoV-2 RBD-ACE2 complex, whereas noscapine has the most efficient compounds in SARS-CoV RBD-ACE2.

Figure 5 reveals that, in case of the squalene, compounds such as RBD-ACE2+carprofen and noscapine, also, CTD-ACE2+ noscapine could be helpful for viral infection treatment of SARS-CoV-2. But acetohexamide and fentanyl have the strongest binding affinity to SARS-CoV RBD-ACE2. For AS01, we found only one compound, hydrocodone, cannot effectively target the binding between RBD/CTD and ACE2 for both of SARS-CoV-2 and SARS-CoV. The compounds identified here could potentially target the binding site between Spike RBD/CTD and ACE2. As suggested by the result, authors believe that these compounds are inhibitors of ACE2 driven virus infections.

The binding energy is due to the energy contributions of all different amino acids and residues around the cavity of target protein on interaction site with the screened molecules. Energy contributions of these 
residues are due to different interactions like hydrogen bonding, van der Waals, electrostatic interactions, $\pi-\pi$ stacking, etc. [62]. As the binding of the S protein to ACE2 is undesirable, it is preferable to diminish the ligand-interface interactions that may bridge, and therefore stabilize, the interaction between the $S$ protein and the ACE2 receptor. The detail of RBD/CTD-ACE2 interface binding to narcotics compounds, i.e., narcotine hemiacetal, codeine, carprofen and noscapine mixed with adjuvants (mannide monooleate, muramyl dipeptide and squalene) were evaluated (Figures 6-8 and S2-S4).

As shown in Figures 6-8 the binding of adjuvants in the active pocket of RBD/CTD-ACE2 were compared to SARS-CoV RBD-ACE2. Also, the binding between S protein and ACE2 with narcotics compounds were compared to SARS-CoV RBD-ACE2 as represented in Figures S2-S4. It seems that variations in the binding free energies occur due to the difference in the hydrophobic interactions and hydrogen bonding formation between RBD/CTD-ACE2's amino acid residues with narcotics compounds and adjuvants.

The system stabilization can occur by lowering the binding free energy for the most stable conformations. To better understand the details of SARS-CoV-2 RBD/CTD-ACE2 interface the binding to narcotine hemiacetal+mannide monooleate were also compared to SARS-CoV RBD-ACE2 as detailed in Table 1. Furthermore, all residues involved in SARS-CoV-2 RBD-ACE2 interface interactions with muramyl dipeptide and carprofen compared to SARS-CoV RBD-ACE2 are summarized in Table 2. According to our results, the SARS-CoV-2 CTD-ACE2 with narcotine hemiacetal+mannide monooleate forms hydrophobic interactions with ten amino acids, which are P258, S257, S254, I256, D615, Y255, P612, Y158, W610 and S611, from the target ACE2 with narcotine hemiacetal and T27, N25, 1123 and A26 of the S protein. Also, 15 residues, P258, S257, W610, S254, D615, Y255, Y158, I256, P612, Y613, P490, V491, L162, S611, and A614 of ACE2 receptor have hydrophobic interactions with mannide monooleate, whereas T27, N25 and 1123 are bound to CTD. There are also, hydrophobic interactions between ten amino acids (e.g., K31, H34, N33, P389, Q288, F390, R393, A387, E37 and K353 of ACE2) and N479, D480, Y481, K390, Y491 and Y442 binding to SARS-CoV RBD for mannide monooleate. Comparison of the contact residues of the SARS-CoV-2 CTD-ACE2 and SARS-CoV RBD, clearly shows that ligand (narcotine hemiacetal+mannide monooleate) binding affinity for the ACE2 receptor in SARS-CoV-2 CTD-ACE2 is greater than that of SARSCoV RBD-ACE2. For narcotine hemiacetal+mannide monooleate, in SARS-CoV-2 CTD-ACE2, we found stronger binding affinity than SAR-CoV RBD.

Table 1: Contact residues at the binding interface of SARS-CoV-2 CTD-ACE2 also involved in interactions with mannide monooleate and narcotine hemiacetal. 


\begin{tabular}{|c|c|c|c|c|}
\hline \multirow[t]{2}{*}{ Targats } & \multicolumn{2}{|c|}{ Mannide monooleate } & \multicolumn{2}{|c|}{ Narcotine hemiacetal } \\
\hline & S protein & ACE2 & S protein & ACE2 \\
\hline $\begin{array}{l}\text { SARS- } \\
\text { CoV-2 } \\
\text { CTD }\end{array}$ & $\begin{array}{l}\text { T27-N25- } \\
\text { I123 }\end{array}$ & $\begin{array}{l}\text { P258-S257-W610-S254-D615- } \\
\text { Y255-Y158-I256-P612-Y613- } \\
\text { P490-V491-L162-S611-A614 }\end{array}$ & $\begin{array}{l}\text { A26-N25- } \\
\text { T27-I123 }\end{array}$ & $\begin{array}{l}\text { P258-S257-S254- } \\
\text { I256-D615-Y255- } \\
\text { P612-Y158-W610- } \\
\text { S611 }\end{array}$ \\
\hline $\begin{array}{l}\text { SARS- } \\
\text { CoV } \\
\text { RBD }\end{array}$ & $\begin{array}{l}\text { N479-D480- } \\
\text { Y481-K390- } \\
\text { Y491-Y442 }\end{array}$ & $\begin{array}{l}\text { K31-H34-N33-P389-Q288-F390- } \\
\text { R393-A387-E37-K353 }\end{array}$ & $\begin{array}{l}\text { Y440-N479- } \\
\text { K390-D 480- } \\
\text { Y481-Y491 }\end{array}$ & $\begin{array}{l}\text { N33-E37-H34-D38- } \\
\text { K353- }\end{array}$ \\
\hline
\end{tabular}

There is some tendency between the hydrophobic bonding groups of the muramyl dipeptide with amino acid residues (i.e., Q91, Y135, D88, D87, K85, Y177, Y187, G178, G98, V99 and S176) to bind to RBD of SARS-CoV-2 and amino acids, like S254, S257, I256, P258, P253, M249 of the ACE2. Also, there are hydrophobic interactions between five amino acids (e.g., S254, P253, S257, M249, P258 and I256 of ACE2) and carprofen. There are also, other residues such as V99, I100, Y135, Q91, S176, D88, Y177, K85 and G178, of SARS-CoV-2 chimeric RBD that are connected with carprofen. For muramyl dipeptide + carprofen, similar to narcotine hemiacetal+mannide monooleate, minor change in SARS-CoV-2 CTD-ACE2 and SAR-CoV RBD connection tendency were observed.

Table 2. Residues at the binding interface of SARS-CoV-2 RBD-ACE2, involved in interactions with muramyl dipeptide and carprofen.

\begin{tabular}{|lllll|}
\hline Targats & Muramyl Dipeptide & & Carprofen & \\
& S protein & ACE2 & S protein & ACE2 \\
\hline $\begin{array}{l}\text { SARS- } \\
\text { CoV-2 }\end{array}$ & Q91-Y135-D88-D87- & S254-S257-I256-P258- & V99-I100-Y135- & S254-P253- \\
RBD & G98-V99-S176 & Q91-S176-D88- & S257-M249- \\
SARS- & Y440-K390-N479-D480- & D30-N33-A387-A386- & Y440-K390-D480- & N33-F390- \\
CoV & Y481-F483-G482-Y491 & R393-Q388-P389-H34- & Y481 & E37-R393- \\
RBD & & E37-K353-E37 & & P389-H34 \\
\hline
\end{tabular}

\section{Conclusions}

In summary, the binding of two crucial active sites of the Spike protein (i.e., RBD and CTD) when complexed to ACE2 receptor was theoretically evaluated using in-silico docking studies. Based on bioinformatics analysis two homology structures of 50 SARS-CoV-2 S proteins were built and were setup for high throughput protein-protein docking studies with ACE2 structures.

The results of the protein-protein docking revealed SARS-CoV-2 S protein (i.e., RBD and CTD) have strong binding affinity toward ACE2. However, this interaction is weaker than that of SARS-CoV S protein. 
Possible mutations in specific loops of S protein, RBD/CTD, might promote binding interaction with the ACE2 receptors. The binding affinity of RBD/CTD when complexed to ACE2 receptor were studied in the presence of accessible natural bioactive alkaloids, i.e., narcotic compounds, via molecular docking. Combination of narcotic compounds with small molecule adjuvants-including mannide monooleate, muramyl dipeptide, squalene and AS01 were found as potential complimentary agents of the ACE2 receptor. The results of the molecular docking revealed a promising binding tendency in narcotic compounds with the ACE2 receptor; to an extent where consequent blocking of ACE2 receptor against SARS-CoV-2 can be optimally achieved.

Combinations of narcotine hemiacetal and mannide monooleate in blocking CTD-ACE2 as well as the combination of carprofen with muramyl dipeptide and squalene in blocking RBD-ACE2 were both shown to be efficient. In conclusion, our results suggest that narcotine hemiacetal, carprofen, codeine and noscapine compounds are capable of interacting with the S protein and ACE2 interface and thus interfere with their binding mechanism through blocking active sites. Our results might have significant applications in considering potential drug candidates in therapeutic treatment of SARS-CoV-2 infection.

\section{Declarations}

\section{Acknowledgements}

We would like to thank Shahid Rajaee Teacher Training University for supporting this work.

\section{Author contributions}

Conceptualization, Esmail Doustkhah and Saeedeh Mohammadi; Data curation, Saeedeh Mohammadi, Nader Sakhaee and Esmail Doustkhah; Investigation, Saeedeh Mohammadi, Ayoub Esmailpour; Software, Saeedeh Mohammadi; Supervision, Ayoub Esmailpour and Esmail Doustkhah; Writing - original draft, Saeedeh Mohammadi; Writing - review \& editing, Nader Sakhaee, Mohammad Esmailpour, and Esmail Doustkhah.

\section{Conflicts of Interest}

The authors declare no conflict of interest.

\section{References}

1. Wang, C., Horby, P. W., Hayden, F. G. \& Gao G.F. A novel coronavirus outbreak of global health concern. Lancet. 395, 470-473 (2020).

2. Li, Q. et al. Early transmission dynamics in Wuhan, China, of novel coronavirus-infected pneumonia. N. Engl. J. Med. 382, 1199-1207 (2020).

3. Huang, C. et al. Clinical features of patients infected with 2019 novel coronavirus in Wuhan, China. Lancet. 395, 497-506 (2020). 
4. Hoffmann, M. et al. SARS-CoV-2 Cell Entry Depends on ACE2 and TMPRSS2 and Is Blocked by a Clinically Proven Protease Inhibitor. Cell. 181, 271-280 (2020).

5. Letko, M., Marzi, A. \& Munster, V. Functional assessment of cell entry and receptor usage for SARSCoV-2 and other lineage B betacoronaviruses. Nat. Microbiol. 5, 562-569 (2020).

6. World Health Organization. 2020. Available online: https://covid19.who.int/ (accessed on 17 February 2021).

7. Kucukoglu, K., Faydalı, N. \& Bul, D. What are the drugs having potential against COVID-19? Med. Chem. Res. 29, 1935-1955 (2020).

8. Gordon, D. E. \& Jang, G. M. et. al., A SARS-CoV-2 protein interaction map reveals targets for drug repurposing. Nature. 583, 459-468 (2020).

9. Sadegh, S. et al. Exploring the SARS-CoV-2 virus-host-drug interactome for drug repurposing. Nat. Commun. 11, 3518 (2020).

10. Zhou, H. et al. Potential therapeutic targets and promising drugs for combating SARS-CoV-2. Br. J. Pharmacol. 177, 3147-3161 (2020).

11. Shyr, Z. A., Gorshkov, K., Chen, C. Z. \& Zheng, W. Drug Discovery Strategies for SARS-CoV-2. J. Pharmacol. Exp. Ther. 375, 127-138 (2020).

12. Komatsu, T. S. et al. Drug binding dynamics of the dimeric SARS-CoV-2 main protease, determined by molecular dynamics simulation. Sci. Rep. 10, 16986 (2020).

13. Li, Q. \& Kang, C. B. Progress in Developing Inhibitors of SARS-CoV-2 3C-Like Protease. Microorganisms. 8, 1250 (2020).

14. Lanjanian, H. et al. SARS-CoV-2 infection susceptibility infuenced by ACE2 genetic polymorphisms: insights fromTehran Cardio-Metabolic Genetic Study. Scientifc Reports. 11, 1529 (2021).

15. Wei, Y., Aris, P., Farookhi, H. \& Xia, X. Predicting mammalian species at risk of being infected by SARS-CoV-2 from an ACE2 perspective. Scientifc Reports. 11, 1702 (2021).

16. Smith, M. D. \& Smith, J. C. Repurposing Therapeutics for COVID-19: Supercomputer-Based Docking to the SARS-CoV-2 Viral Spike Protein and Viral Spike Protein-Human ACE2 Interface. Chem Rxiv. https://doi.org/10.26434/chemrxiv.11871402.v4 (2020).

17. Van Norman, G. A. Drugs, devices, and the FDA: Part 1: An overview of approval processes for drugs. JACC Basic Transl. Sci. 1, 170-179 (2016).

18. Sethi, A. \& Bach, H. Evaluation of Current Therapies for COVID-19 Treatment. Microorganisms. 8, 1097 (2020).

19. Mellon, R. D. \& Bayer, B. M. Evidence for central opioid receptors in the immunomodulatory effects of morphine: review of potential mechanism s of action. J. Neuroimmunol. 83, 19-28 (1998).

20. Masoom Maan, M. A., Siddique, A., Iqbal, R. \& Ahmad Kh. COVID-19 Pandemic and Chronic Pain Management: Impact and suggestions. Pak. Armed. Forces. Med. J. 70, S413-S417 (2020).

21. Damani, A. et al. Approaches and Best Practices for Managing Cancer Pain within the Constraints of the COVID-19 Pandemic in India. Indian. J. Palliat. Care. 26, S106-S115 (2020). 
22. Datarkar, A., Purohit, S., Taya, S. \& Bhawalkar, A. Operating Room Protocols in OMFS During Corona virus (Covid-19) Pandemic. J. Maxillofac. Oral Surg. 19 (3), 327-331 (2020).

23. Lee, S., Meyler, P., Mozel, M., Tauh, T. \& Merchant, R. Asymptomatic carriage and transmission of SARS-CoV-2: What do we know? Patients asymptomatiques du SARS-CoV-2 et transmission du virus: Ou`en sont nos connaissances? Can J Anesth/J Can Anesth. https://doi.org/10.1007/s12630-02001729-x.

24. Huang, J. et al. Recommendation about the perioperative prevention of infection to healthcare workers and the anesthesia management of children with SARSCoV-2 infection. World Jnl. Ped. Surgery. 3, e000126 (2020).

25. Aminnejad, R., Salimi, A. \& Saeidi, M. Lidocaine during intubation and extubation in patients with coronavirus disease (COVID-19). Can J Anesth/J Can Anesth. 67, 759 (2020).

26. dos Santos, S. et al. L.N. Pituitary tumor resection in a patient with SARS-CoV-2 (COVID-19) infection. A case report and suggested airway management guidelines. Rev. Bras. Anestesiol. 70 (2), 165-170 (2020).

27. Koutroumanidis, M. et al. Alpha coma EEG pattern in patients with severe COVID-19 related encephalopathy. Clin. Neurophysiol. 132, 218-225 (2020).

28. Farsalinos, K., Barbouni, A., Niaura, R. \& Smoking Vaping and Hospitalization for COVID-19, Qeios ID: Z6908A.2 2020, https://doi.org/10.32388/Z6908A.2.

29. Farsalinos, K., Barbouni, A., Niaura, R. \& Smoking Vaping and Hospitalization for COVID-19, Qeios ID: Z6908A.11 2020, https://doi.org/10.32388/Z6908A.11.

30. Farsalinos, K., Barbouni, A. \& Niaura, R. Systematic review of the prevalence of current smoking among hospitalized COVID-19 patients in China: Could nicotine be a therapeutic option? Int. Emerg. Med. 15, 845-852 (2020).

31. Mohammadi, S. et al. In silico Investigation on the Inhibiting Role of Nicotine/Caffeine by Blocking the S Protein of SARS-CoV-2 Versus ACE2 Receptor. Microorganisms. 8, 1600 (2020).

32. Mckee, A. S., Macleod, M. K. L., Kappler, J. W. \& Marrack, P. Immune mechanisms of protection: Can adjuvants rise to the challenge? BMC Biol. 8, 37 (2010).

33. Flower, D. R. \& Perrie, Y. Immunomic Discovery of Adjuvants and Candidate Subunit Vaccines. Springer Science New York 2013. DOI 10.1007/978-1-4614-5070-5072.

34. Flower, D. R. Systematic identification of small molecule adjuvants. Expert Opin. Drug Discov. 7 (9), 807-817 (2012).

35. Wang, Z. B. \& Xu, J. Better Adjuvants for Better Vaccines: Progress in Adjuvant Delivery Systems, Modifications, and Adjuvant-Antigen Codelivery. Vaccines. 8, 128 (2020).

36. Aucouturier, J., Ascarateil, S. \& Dupuis, L. The use of oil adjuvants in therapeutic vaccines. Vaccine. 24, 44-45 (2006).

37. Miles, A. P. et al. Montanide ${ }^{\circledR}$ ISA 720 vaccines: quality control of emulsions, stability of formulated antigens, and comparative immunogenicity of vaccine formulations. Vaccine. 23, 2530-2539 
(2005).

38. Benferhat, R., Martinon, F., Krust, B., Le Grand, R. \& Hovanessian, A. G. The CBD1 peptide corresponding to the caveolin-1 binding domain of HIV-1 glycoprotein gp41 elicits neutralizing antibodies in cynomolgus macaques when administered with the tetanus T helper epitope. Mol. Immunol. 46, 705-712 (2009).

39. Peter, K., Men, Y., Pantaleo, G., Gander, B. \& Corradin, G. Induction of a cytotoxic T-cell response to HIV-1 proteins with short synthetic peptides and human compatible adjuvants. Vaccine. 19, 41214129 (2001).

40. Toledo, H. et al. A phase I clinical trial of a multi-epitope polypeptide TAB9 combined with Montanide ISA 720 adjuvant in non-HIV-1 infected human volunteers. Vaccine. 19, 4328-4336 (2001).

41. Zhu, D. et al. Long term stability of a recombinant Plasmodium falciparum AMA1 malaria vaccine adjuvanted with Montanide® ISA 720 and stabilized with glycine. Vaccine. 29, 3640-3645 (2011).

42. McCarthy, J. S. et al. A Phase 1 Trial of MSP2-C1, a Blood-Stage Malaria Vaccine Containing 2 Isoforms of MSP2 Formulated with Montanide H ISA 720. PLoS ONE. 6, e24413 (2011).

43. Oliveira, G. A., Wetzel, K., Calvo-Calle, J. M., Nussenzweig, R. \& Schmidt, A. et al, Safety and Enhanced Immunogenicity of a Hepatitis B Core Particle Plasmodium falciparum Malaria Vaccine Formulated in Adjuvant Montanide ISA 720 in a Phase I Trial. Infect. Immun. 73, 3587-3597 (2005).

44. Azmi, F., Al, H. A., Fuaad, A., Skwarczynski, M. \& Toth, I. Recent progress in adjuvant discovery for peptidebased subunit vaccines. Hum. Vaccin. Immunother. 10, 778-796 (2014).

45. Meshcheryakova, E., Makarov, E., Philpott, D., Andronova, T. \& Ivanov, V. Evidence for correlation between the intensities of adjuvant effects and NOD2 activation by monomeric, dimeric and lipophylic derivatives of $\mathrm{N}$-acetylglucosaminyl-Nacetylmuramyl peptides. Vaccine. 25, 4515-4520 (2007).

46. Chen, Y. Z. et al. Identification of SARS-COV Spike Protein-Derived and HLA-A2-Restricted Human CTL Epitopes by Using a New Muramyl Dipeptide-Derivative Adjuvant. Int. J. Immunopathol. Pharmacol. 23, 165-177 (2010).

47. Robson, B. COVID-19 Coronavirus spike protein analysis for synthetic vaccines, a peptidomimetic antagonist, and therapeutic drugs, and analysis of a proposed achilles' heel conserved region to minimize probability of escape mutations and drug resistance. Comput. Biol. Med. 121, 103749 (2010).

48. Waterhouse, A. et al. SWISS-MODEL: homology modelling of protein structures and complexes. Nucleic Acids Res. 46, 296-303 (2018).

49. Hall, T. A. \& BioEdit A user-friendly biological sequence alignment editor and analysis program for Windows 95/98/NT. Nucleic Acids Symp. Ser. 41, 95-98(1999).

50. Thompson, J. D., Gibson, T. J. \& Higgins, D. G. Multiple sequence alignment using ClustalW and ClustalX. Current protocols. Bioinform. 1, 2-3 (2003).

51. Kumar, S., Stecher, G., Li, M., Knyaz, C. \& Tamura, K. MEGA X: Molecular Evolutionary Genetics Analysis across computing platforms. Mol. Biol. Evol. 35, 1547-1549 (2018). 
52. Waterhouse, A. et al. SWISS-MODEL: homology modelling of protein structures and complexes, Nucleic Acids Res. 46(W1), W296-W303(2018).

53. Johansson, M. U., Zoete, V., Michielin, O. \& Guex, N. Defining and searching for structural motifs using DeepView/Swiss-PdbViewer. BMC Bioinformatics. 13, 173 (2012).

54. Schrödinger, L. L. C. The PyMOL molecular graphics system version 1.8, (2015).

55. Alonso, H. A., Bliznyuk, A. A. \& Gready, J. E. Combining docking and molecular dynamic simulations in drug design. Med. Res. Rev. 26, 531-568 (2006).

56. Morris, G. M. et al. AutoDock4 and AutoDockTools4: Automated docking with selective receptor flexibility. J. Comput. Chem. 30, 2785-27891 (2009).

57. Morris, G. M. et al. Automated docking using a Lamarckian genetic algorithm and an empirical binding free energy function. J. Comput. Chem. 19, 1639-1662 (1998).

58. Audipudi, A. V., Badri, R. R. \& Bhaskar, C. V. S. Silico Molecular Docking Analysis of Secondary Metabolites Present in Leaf Extract of Cassia occidentalis Linn; Medicinal Plants:

BiodiversityGermany, 501-508(Sustainable Utilization and Conservation; Springer, Berlin/Heidelberg, 2020). GC-MS and In

59. Holland, J. H. Adaptation in Natural and Artificial Systems (University of Michigan Press, Ann Arbor, MI, USA, 1975).

60. Abagyan, R., Totrov, M. \& Kuznetsov, D. ICM-A new method for protein modeling and design: applications to docking and structure prediction from the distorted native conformation. J. Comput. Chem. 15, 488-506 (1994).

61. Wu, C. et al. Analysis of therapeutic targets for SARS-CoV-2 and discovery of potential drugs by computational methods. Acta Pharm. Sin. B. 10, 766-788 (2020).

62. Kumara, D. et al. Understanding the binding affinity of noscapines with protease of SARS-CoV-2 for COVID-19 using MD simulations at different temperatures. J. Biomol. Struct. Dyn. 4, 1-14 (2020).

\section{Figures}


<smiles>CC(=O)Nc1ccc(O)cc1</smiles>

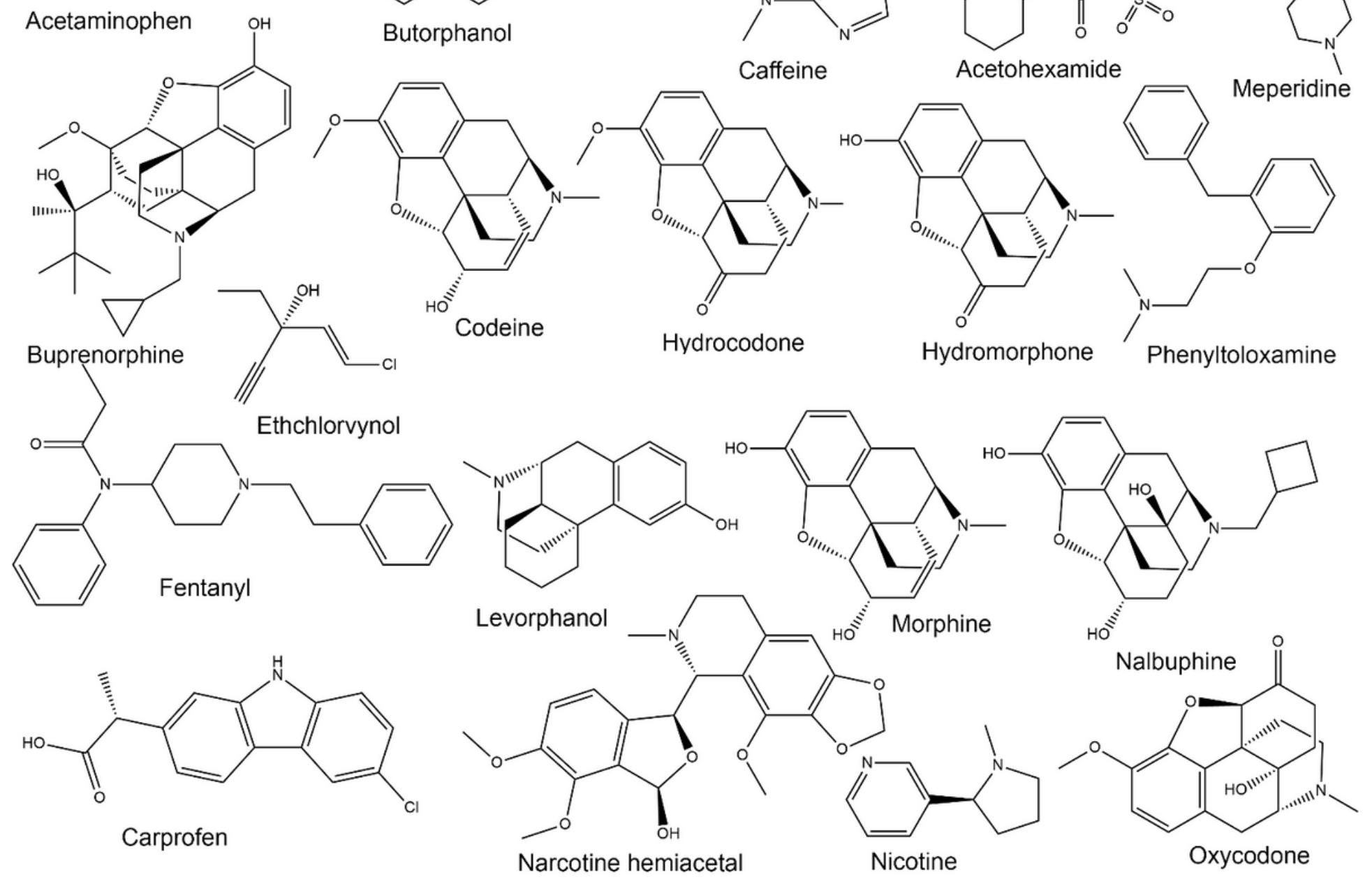

Figure 1

The phytochemical structures of narcotic compounds studied. 


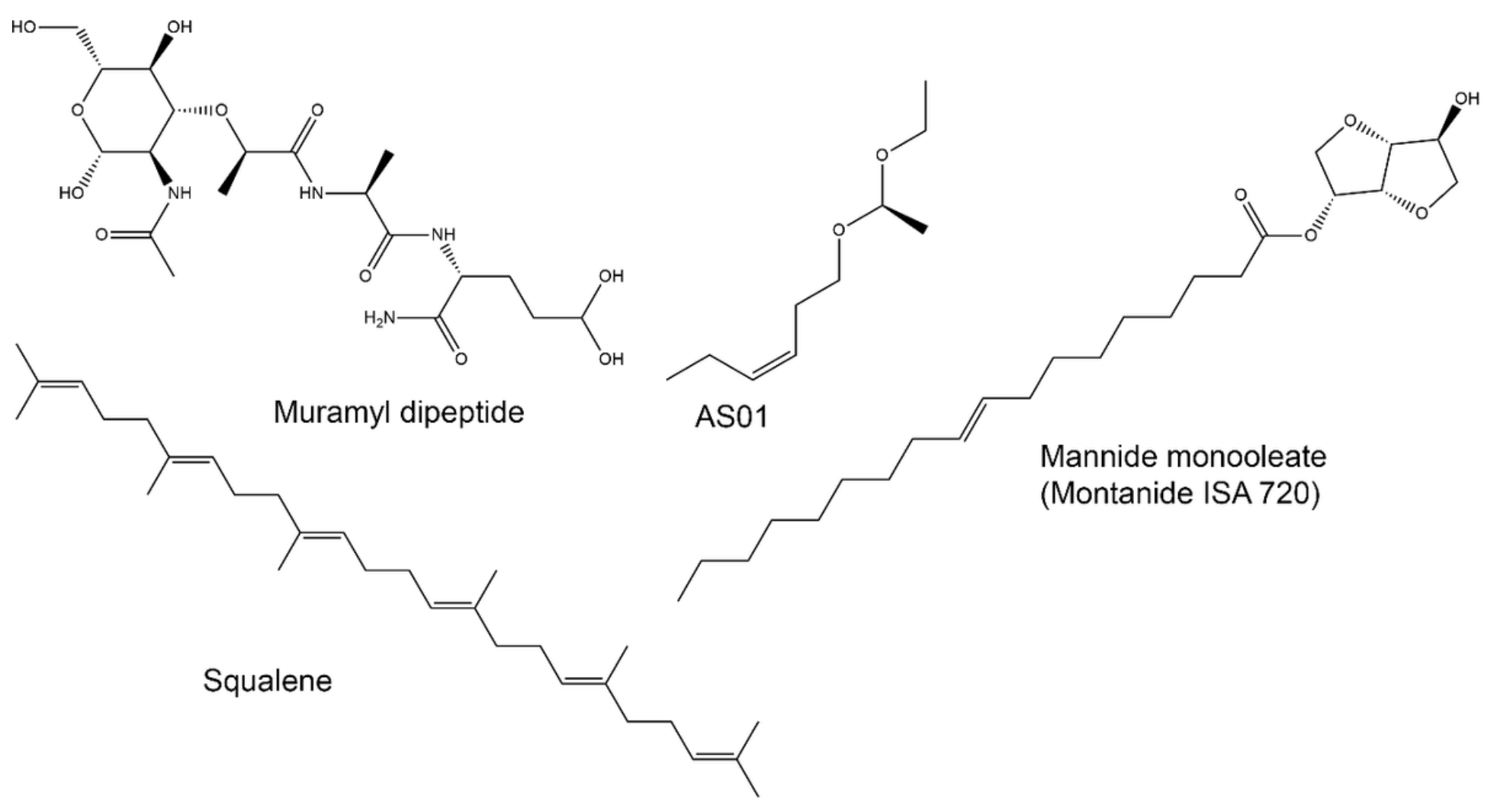

Figure 2

Small molecule adjuvants of natural and synthetic origin (continued). 

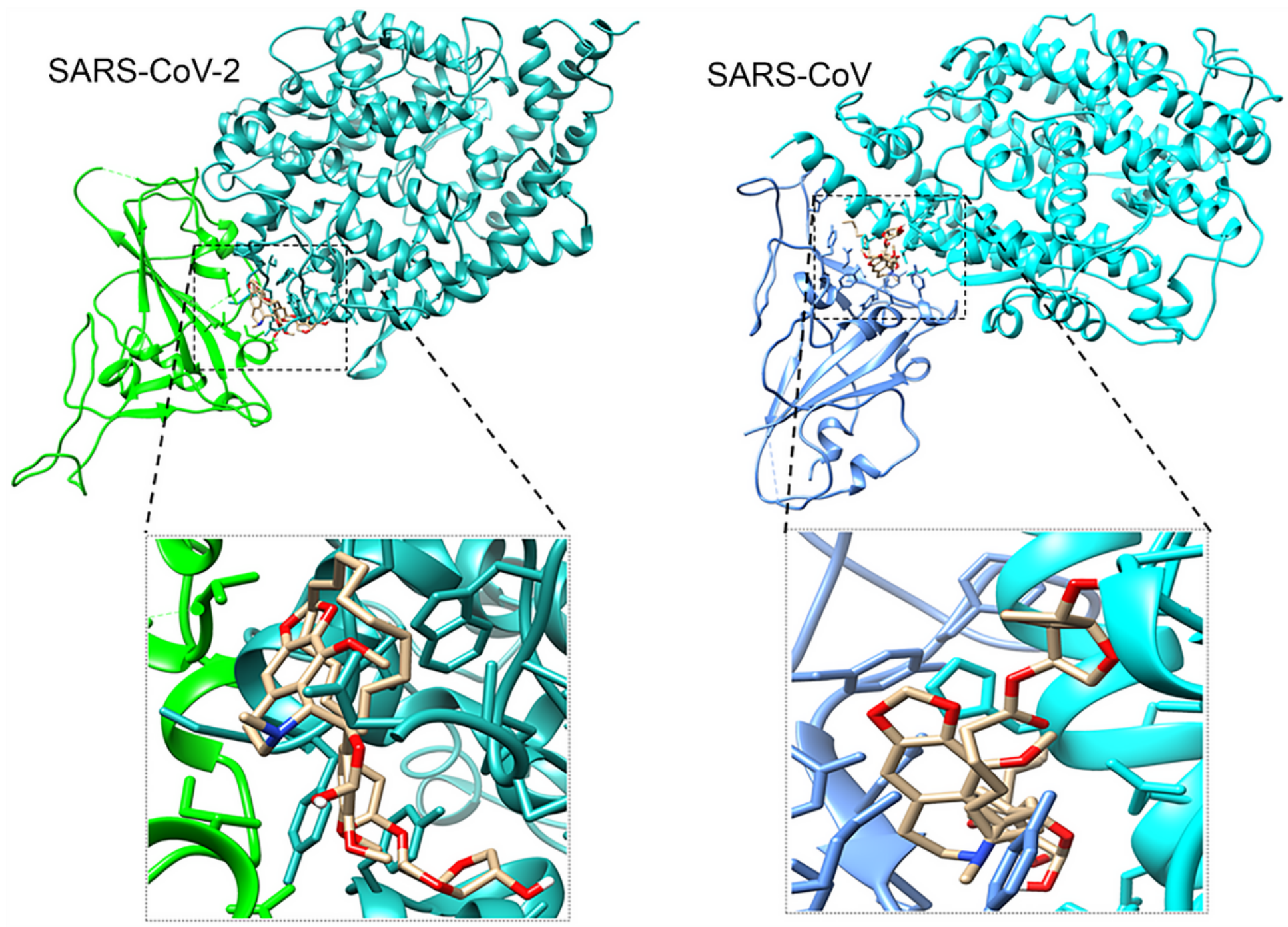

Figure 3

Interactions between ACE2/SARS-CoV-2-CTD and SARS-CoV RBD-ACE2 complexes with narcotic compounds and adjuvants. 

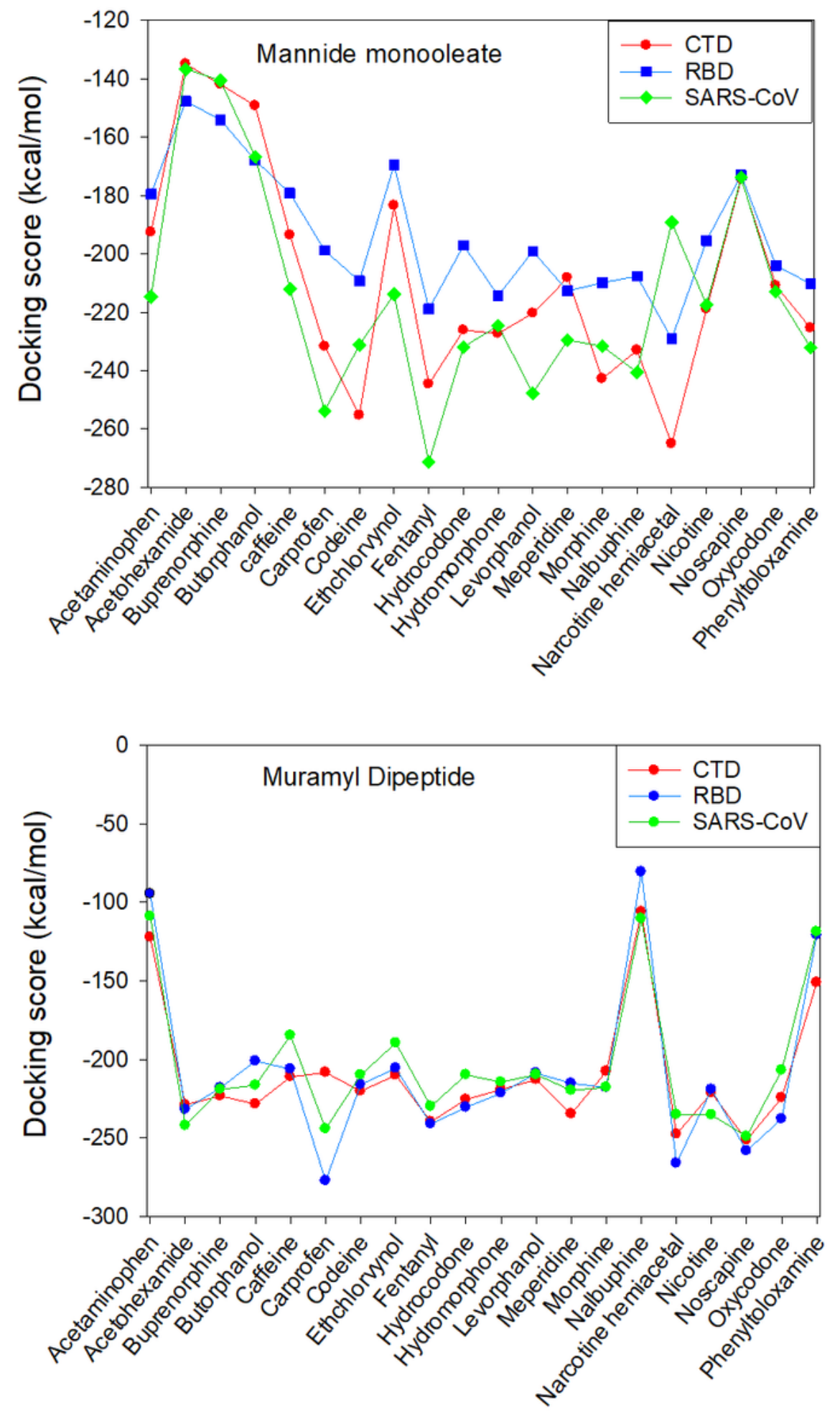

Figure 4

The docking score for narcotic compounds+mannide monooleate and muramyl dipeptide with SARSCoV-2 CTD/RBD and SARS-CoV RBD bound to ACE2. 

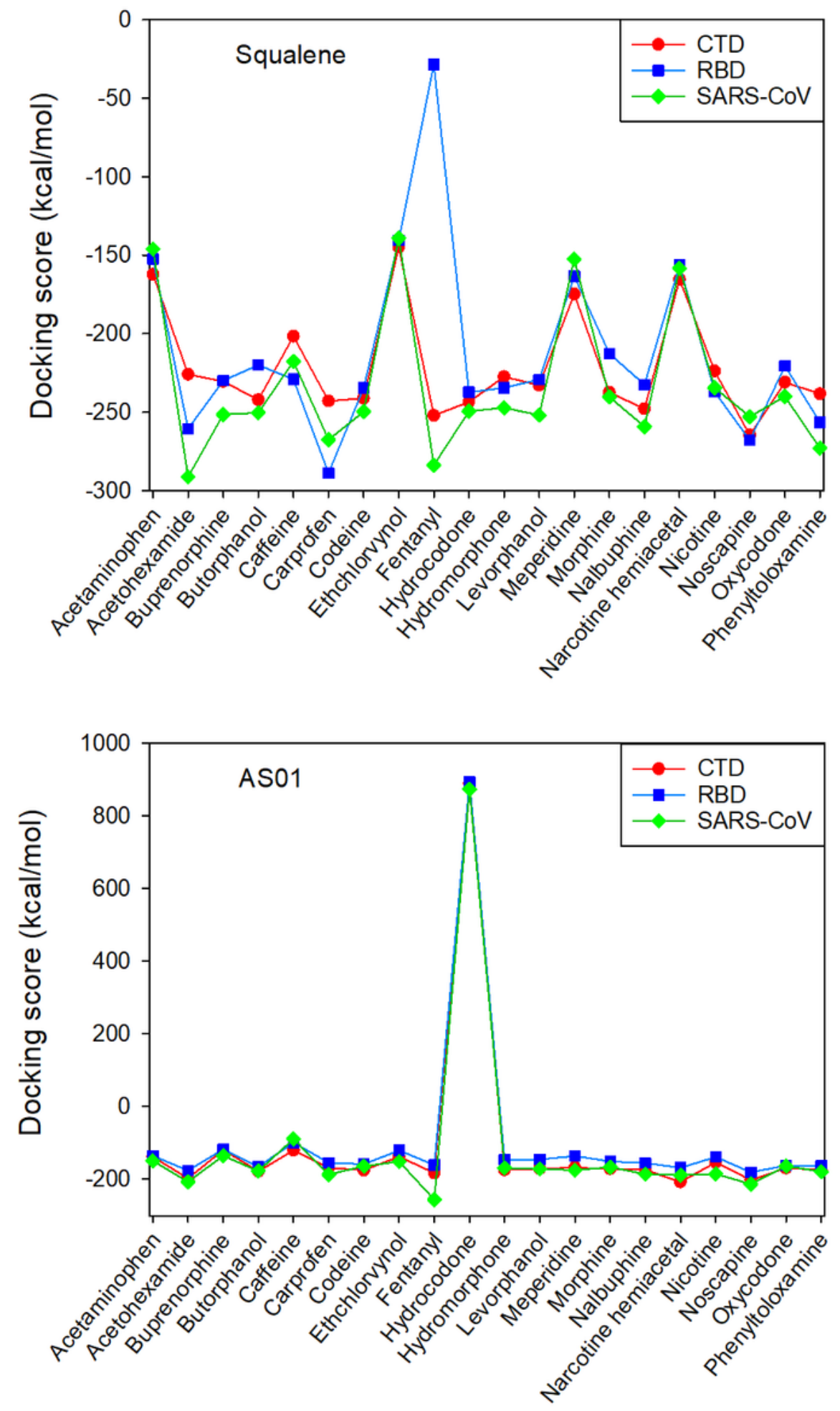

Figure 5

The docking score for narcotic compounds+squalene and AS01 with SARS-CoV-2 CTD/RBD and SARSCoV RBD bound to ACE2. 


\section{Mannide monooleate}

SARS-CoV-2 CTD+Codeine

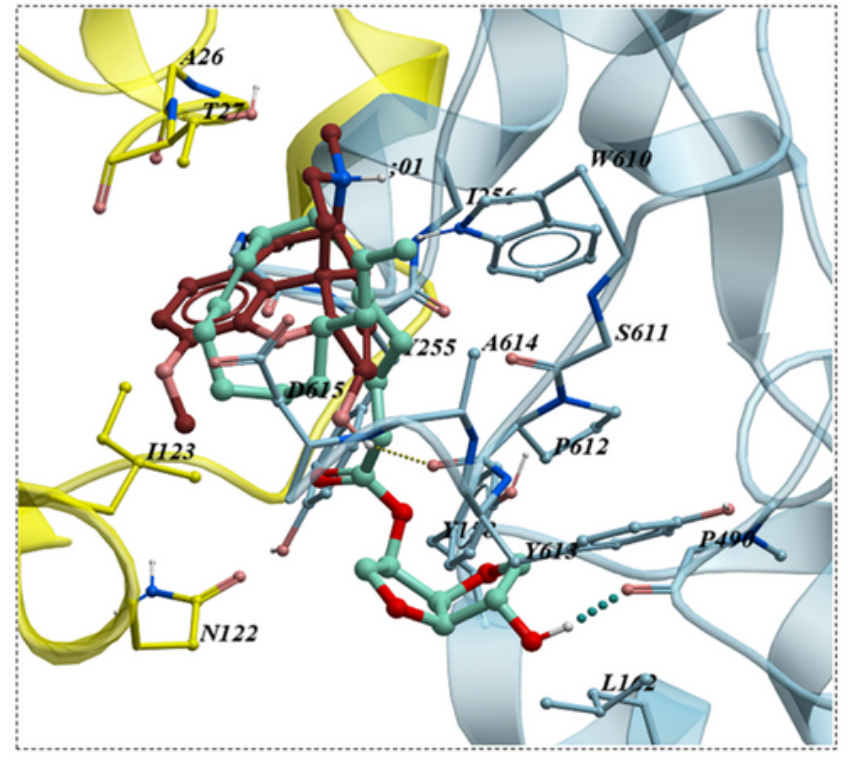

SARS-CoV-2 CTD+Narcotine hemiacetal

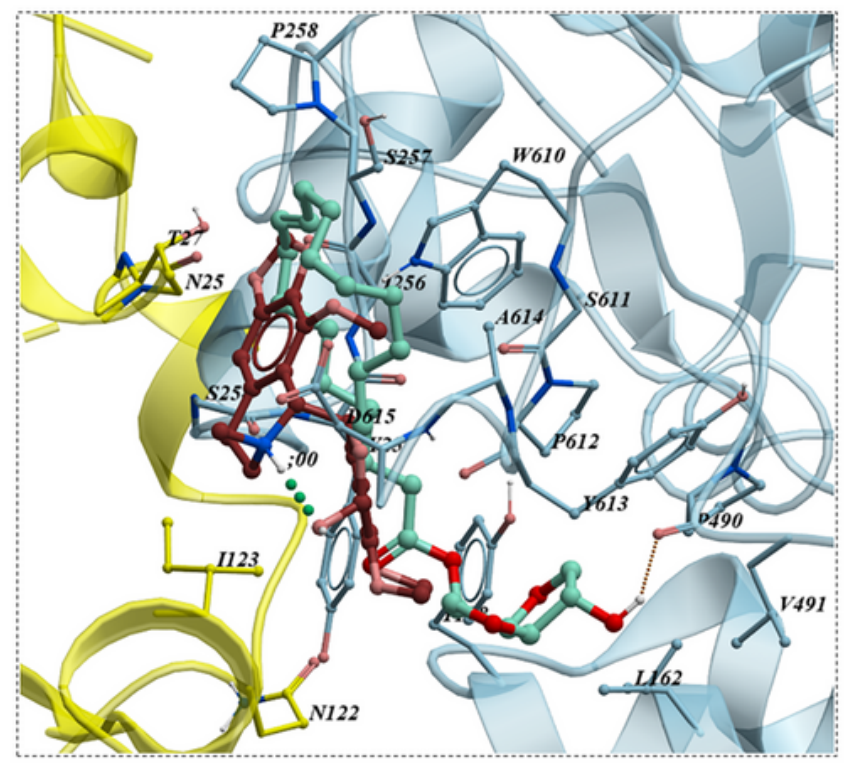

SARS-CoV RBD+Codeine

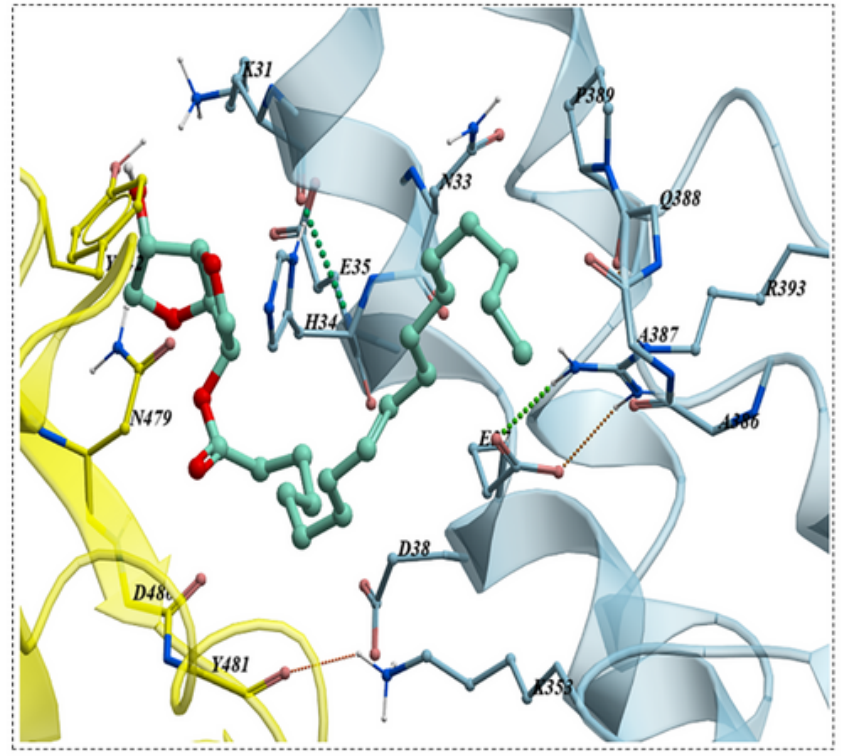

\section{SARS-CoV RBD+Narcotine hemiacetal}

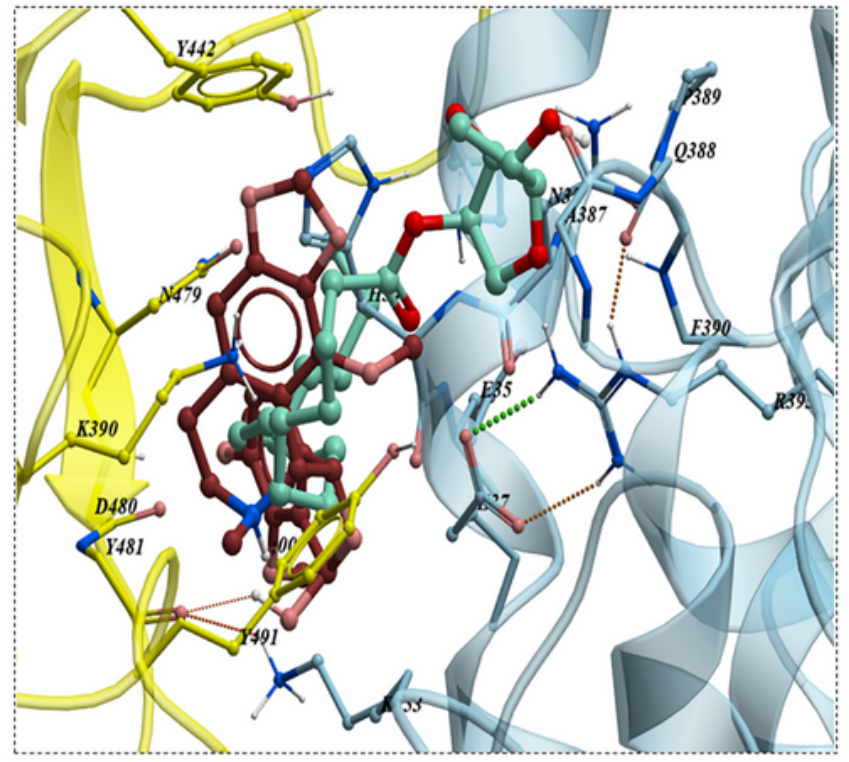

Figure 6

Binding interface structure of SARS-CoV-2 CTD and human ACE2 with narcotine hemiacetal and codeine + mannide monooleate (in pocket of mannide monooleate). Compare to SARS-CoV RBD. ACE2 is in blue, the core of CTD is in yellow. 


\section{Muramyl Dipeptede}

\section{SARS-CoV-2 RBD+Carprofen}

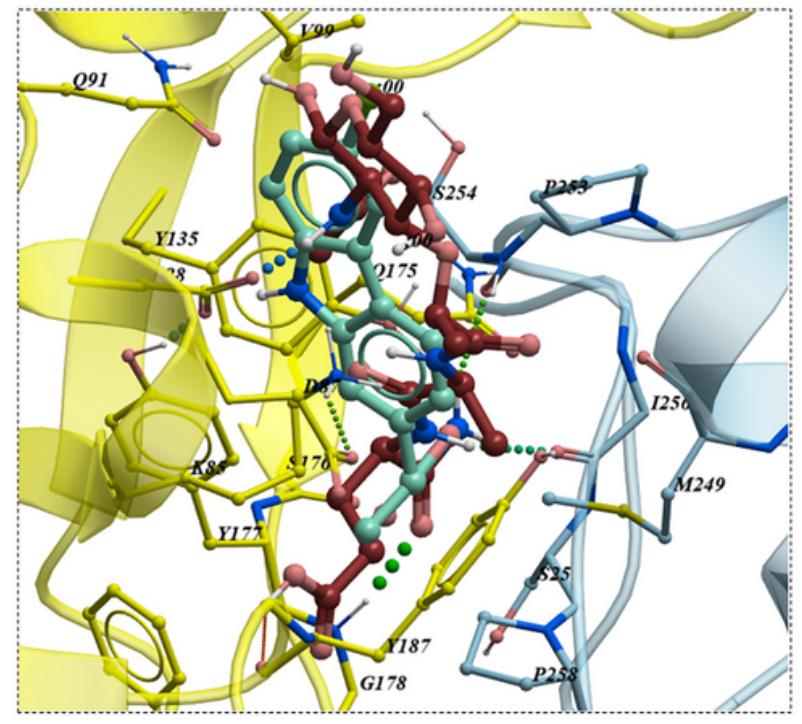

SARS-CoV-2 RBD+Narcotine hemiacetal

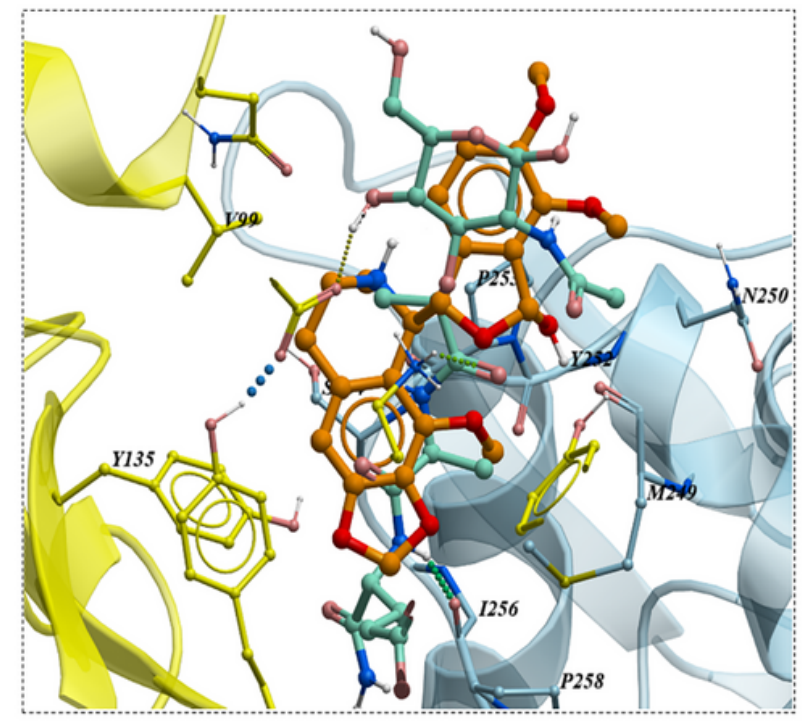

SARS-CoV RBD+Carprofen

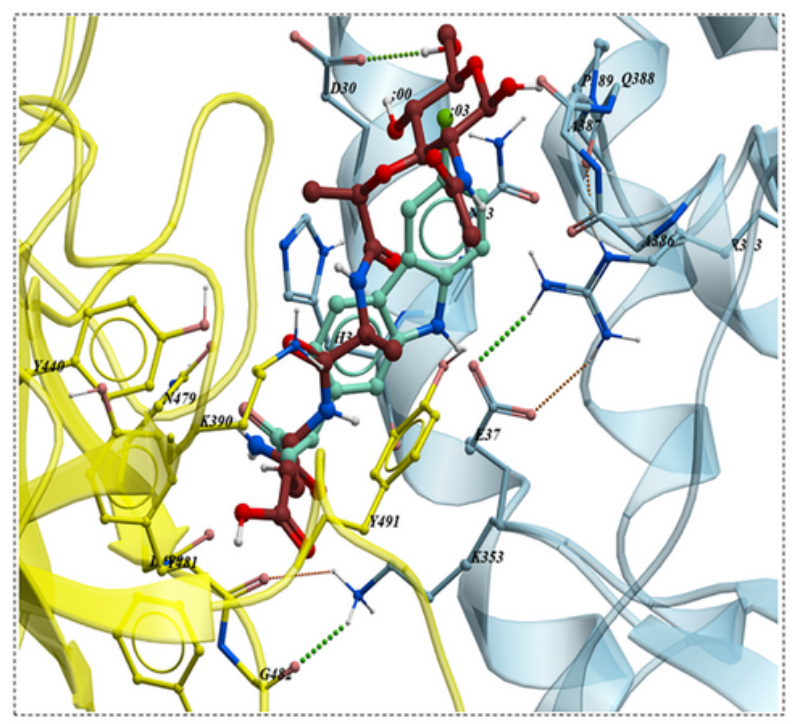

SARS-CoV RBD+Narcotine hemiacetal

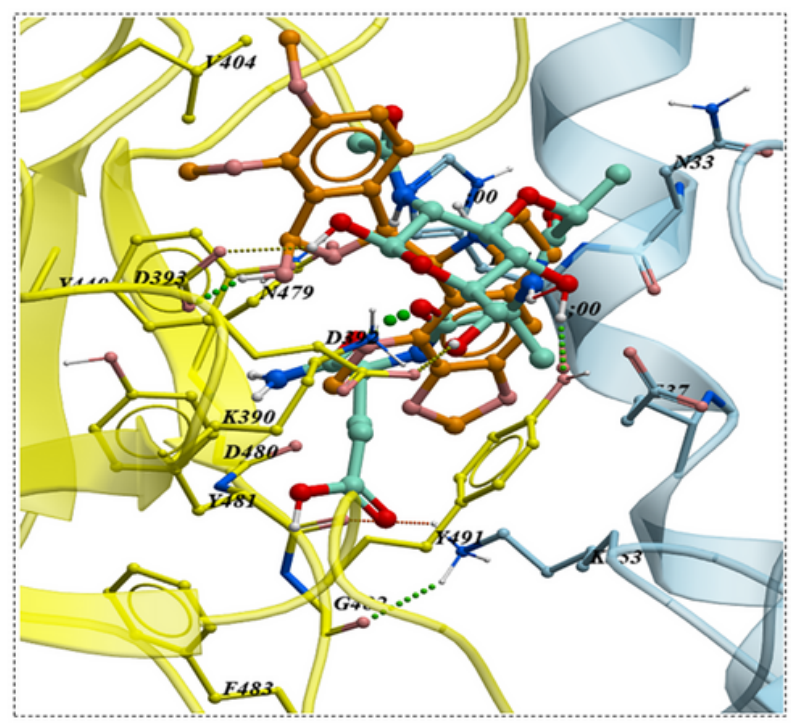

Figure 7

The binding interface of RBD (yellow) external subdomain of spike glycoprotein with receptor ACE2 (blue) in complex of muramyl dipeptide + carprofen and narcotine hemiacetal. Compare to SARS-CoV RBD in pocket of muramyl dipeptide. 


\section{Squalene}

SARS-CoV-2 CTD+Noscapine

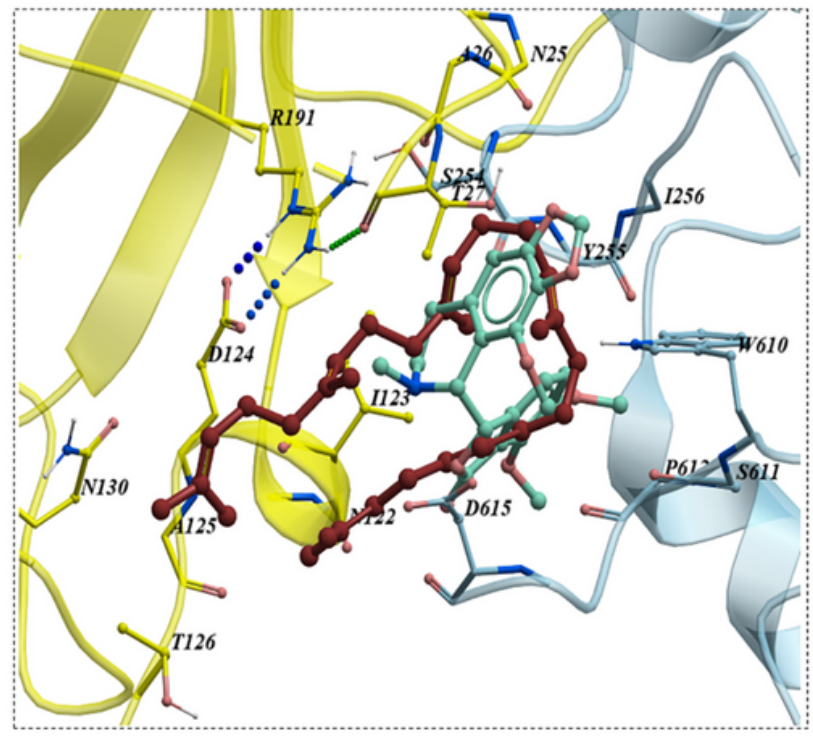

\section{SARS-CoV-2 RBD+Carprofen}

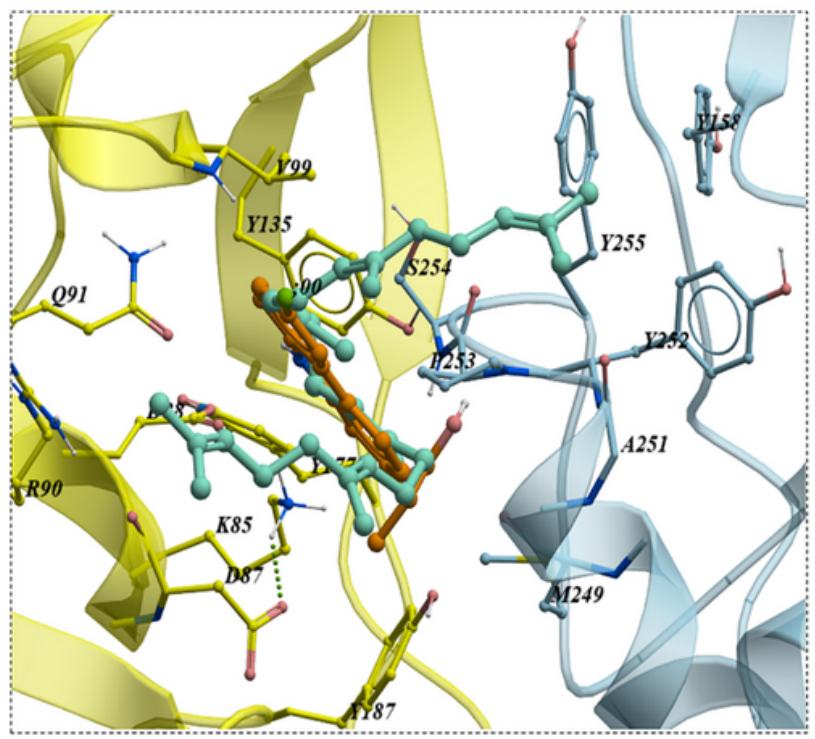

SARS-CoV RBD+Noscapine

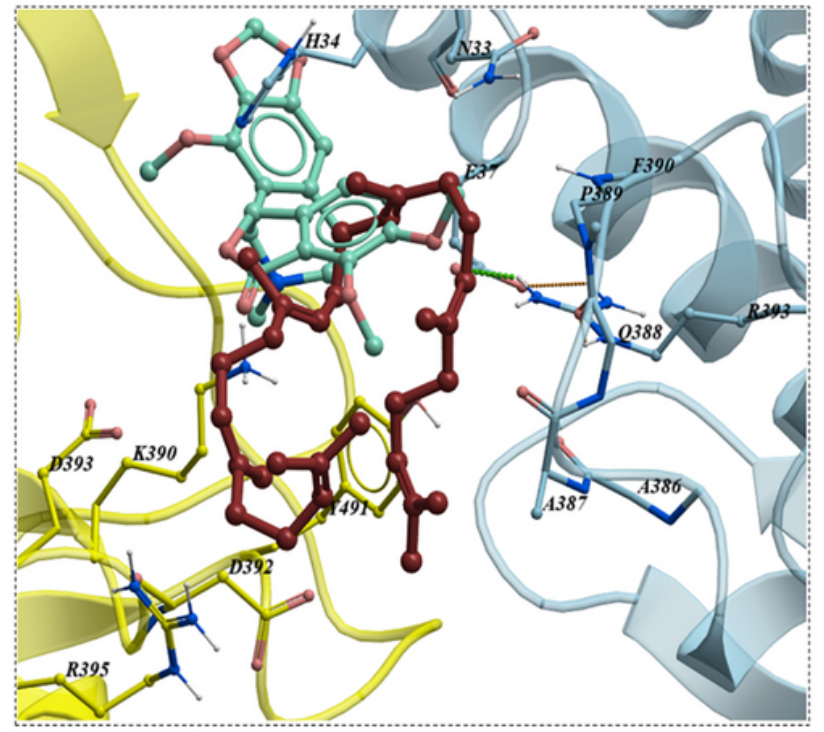

SARS-CoV RBD+Carprofen

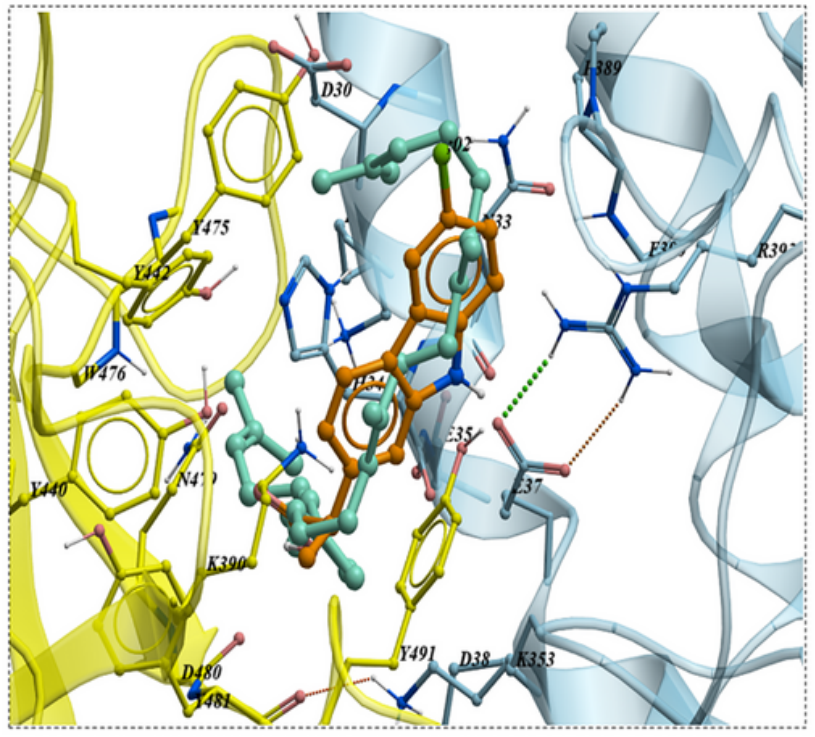

Figure 8

The binding interface of RBD/CTD (yellow) in spike glycoprotein with receptor ACE2 (blue) in complex of squalene + carprofen and nocapine. Compare to SARS-CoV RBD in pocket of squalene.

\section{Supplementary Files}

This is a list of supplementary files associated with this preprint. Click to download. 
- SI.docx

Page 22/22 